\title{
High Impact Practices-Detecting and managing hypertension in the extended postnatal period: Preventing postnatal pre- eclampsia and eclampsia
}

Charlotte E. Warren

Population Council

Follow this and additional works at: https://knowledgecommons.popcouncil.org/departments_sbsr-rh

Part of the Maternal and Child Health Commons

How does access to this work benefit you? Let us know!

\section{Recommended Citation}

Warren, Charlotte E. 2020. "High Impact Practices-Detecting and managing hypertension in the extended postnatal period: Preventing postnatal pre-eclampsia and eclampsia," Hypertensive Disorders in Pregnancy brief. Washington, DC: Population Council. 


\section{HIGH IMPACT PRACTICES}

\section{Detecting and managing hypertension in the extended}

\section{postnatal period}

\section{Preventing postnatal pre-eclampsia and eclampsia}

\section{WHAT IS THE HIGH-IMPACT PRACTICE IN MATERNAL HEALTH?}

Measure every woman's blood pressure after birth, daily until discharge and on each contact in the extended postnatal period. (The extended postnatal period is defined as 12 months following a birth).

All women should stay at a health facility for at least 24 hours after birth. If their blood pressure (BP) was raised, then women should stay until their BP and other vital signs are normal and treated or referred [1].

If women experienced a hypertensive disorder during pregnancy or early postnatal period - regular checkups (BP measurement) are required. Specifically at a minimum: on Day 1 and once between Day 3 and 5 when the risk of adverse cardiovascular event is high [1, 2]; again, at the 6 week and 3-month postnatal check, and when infants are brought for immunization, and at any other contact with the health system. Appropriate treatment of hypertension postnatally is essential to prevent long-term maternal morbidity.

\section{BACKGROUND}

Postnatal hypertension is most often caused by high BP from pre-eclampsia and gestational hypertension that continues after delivery. Pre-eclampsia may develop for the first time after childbirth within the first 3-5 days but may occur up to 4 weeks postnatal. Evidence suggests that about $25 \%$ of women with hypertensive disorders in pregnancy (HDPs) experience a deterioration of end organ function postnatally (period spanning 6-8 weeks after delivery).[4] Close BP monitoring after childbirth is highly recommended, as $20-44 \%$ of maternal deaths occur in low resource settings after childbirth [1, 5-9]. In the early

\section{KEY POINTS}

- The extended postnatal period provides a "window of opportunity" to monitor a woman's BP and to check for persistent hypertension. Often women are asymptomatic - meaning they are not aware that their BP is high.

- If BP is measured routinely at any contact with health system during the extended postnatal period hypertension is more likely to be detected and managed appropriately.

- Women with pre-eclampsia have a 3.7-fold increased risk of future hypertension, 2.2-fold risk of ischemic heart disease, and 1.8-fold risk of stroke [3]

- Measure every woman's BP after birth, daily until discharge. At a minimum on Day 1 and between Days 3-5 after birth if discharged early).

- If women continue to have high BP postnatally, they must receive hypertensive medicines with close follow-up [1,2].

- Women who were previously normotensive during pregnancy, may develop hypertension or preeclampsia for the first time after delivery.

postnatal period, women with pre-existing pre-eclampsia who are hospitalized should be considered at high risk for complications for at least 3-5 days and should have their BP and clinical condition monitored closely [1, 8].

Women with early onset pre-eclampsia (< 34 weeks) and gestational hypertension are at higher risk of developing persistent cardiovascular impairment 12 months after delivery [4]. Pre-eclampsia occurring early in pregnancy or presenting in its severe form or persistence of proteinuria
The Population Council conducts research and delivers solutions that improve lives around the world. Big ideas supported by evidence:

It's our model for global change. popcouncil.org

(C) 2020 The Population Council, Inc.

\section{Evowa Eclampsia}


beyond three to six months postnatally suggests possible chronic hypertension or renal disease. The risk of chronic diseases such as hypertension, ischemic heart disease, stroke, renal disease, and diabetes mellitus increases in women with pre-eclampsia before 37 weeks or have had placental abruption or stillbirth [3]. Higher BMI and history of diabetes mellitus is associated with postnatal hypertension. It is possible that postnatal hypertension represents sub-clinical/asymptomatic or unresolved hypertension [10].

Women who have had gestational hypertension or preeclampsia should receive a comprehensive medical review within six to eight weeks after delivery, and at other contacts with the health system for up to 12 months, as well as discuss increased lifetime cardiovascular risk, anti-hypertensive treatment, and risk in future pregnancy $[5,8]$.

Detecting, and/or managing unresolved BP during postnatal period is one of several promising high impact practices in maternal health. HIPs are identified by a technical working group (TWG) of international experts. This promising practice has limited evidence, with more information needed to fully document implementation experience and practice. The TWG advises that these practices be promoted widely, provided that they are implemented within the context of research and are carefully evaluated in terms of both process and impact. For more information about other HIPs, see https://www. popcouncil.org/research/ending-eclampsia1.

\section{WHY IS THIS PRACTICE IMPORTANT?}

Hypertension is a leading cause of admission postnatally. Early detection prevents complications. Between $30 \%$ and $40 \%$ of women who experience eclampsia occurs in the postnatal period [11]. In addition to using antihypertensives to control high BP, severe pre-eclampsia and eclampsia (seizures) are managed using magnesium sulphate $\left(\mathrm{MgSO}_{4}\right)[1,2,8]$.

Hypertension that appears for the first time postnatally does so most commonly on days 3-6. Hypertension should be recognized and effectively treated to prevent increases in severity and to avoid unnecessary delays in discharge.

A third of women who have HDPs will have sustained hypertension postnatally, although often their BP may seem normal in the early postnatal period $[12,13]$.
Definitions: Hypertensive disorders of pregnancy

Pre-eclampsia and Eclampsia

- Pre-eclampsia: Onset of a new episode of hypertension after 20 weeks of pregnancy or postpartum, characterized by: persistent hypertension (systolic BP $\geq 140 \mathrm{~mm}$ Hg diastolic BP $\geq 90 \mathrm{~mm} \mathrm{Hg}$ )and substantial proteinuria $(>0.3$ $\mathrm{g} / 24$ hours) or any biochemical or hematological abnormality.

- Severe pre-eclampsia is pre-eclampsia with severe signs and symptoms(severe headache, upper abdominal pain, blurred vision, decreased urine output)

- Eclampsia: Generalized seizures, in addition to pre-eclampsia criteria

- Gestational hypertension: hypertension $(\geq 140 / \geq 90$ $\mathrm{mm} \mathrm{Hg}$ ) after 20 weeks of pregnancy without proteinuria or any biochemical or hematological abnormality

- Chronic hypertension: pre-existing high BP ( $\geq 140$ $\mathrm{mmHg}$ systolic or $\geq 90 \mathrm{mmHg}$ ) before the onset of pregnancy or diagnosed in the first 20 weeks of gestation

- Hypertension: BP of $\geq 140 \mathrm{mmHg}$ systolic and/or $\geq$ $90 \mathrm{mmHg}$ diastolic

- Severe hypertension: BP $\geq 160 \mathrm{mmHg}$ systolic or $\geq 110 \mathrm{mmHg}$ diastolic

Appropriate treatment of postnatal hypertension is essential to prevent maternal morbidity and mortality from cerebral hemorrhage $[12,13]$.

Hypertensive disorders of pregnancy and the postnatal period are associated with several long-term complications, including potential complications in future pregnancies and a high lifetime risk of cardiovascular disease (chronic hypertension, ischemic heart disease, stroke, dementia) $[1,3]$. 


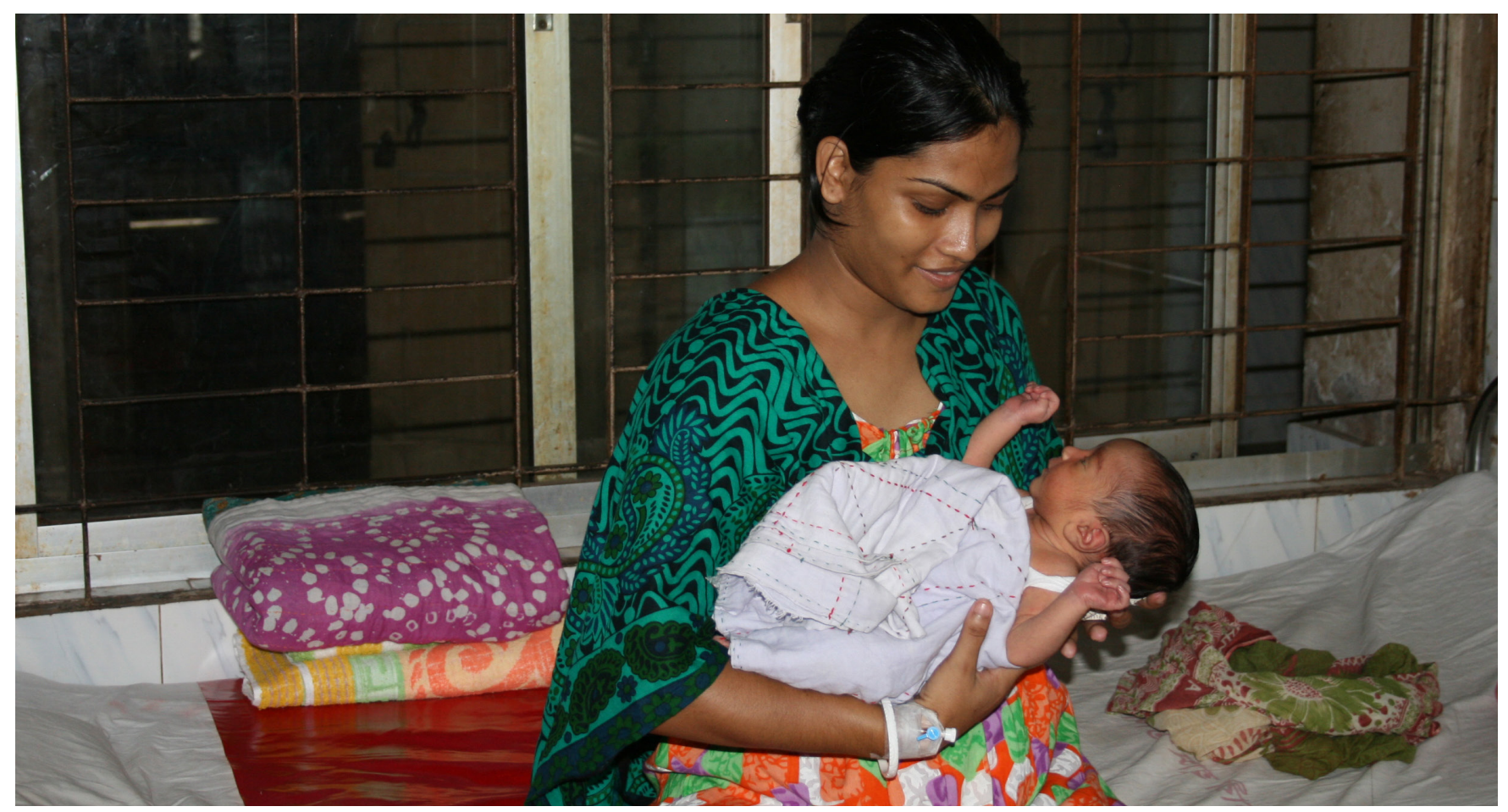

\section{CHALLENGES OF ADHERING TO RECOMMENDATIONS TO MONITOR BP IN POSTNATAL PERIOD}

The current World Health Organization (WHO) PNC recommendations include close follow up on Day 1 and 3, within 1 to 2 weeks and again at 6 weeks [14]. However, the short time women stay post-delivery in health facilities and the poor quality of postnatal contacts in many countries reflect the lack of importance given to comprehensive PNC [9].

In Nigeria, the national clinical guidelines recommend daily BP measurement for the first 3 days after delivery for women who suffered from HPD, and at least once between days 3-5. In a cohort of 406 women who had experienced HPD in eight tertiary health facilities in Nigeria, 99\% of women had a BP check on the day after childbirth. However, adherence progressively declined over the next four days, and by the fifth day postnatal, BP was only checked in 38\% of women [15]. In a similar study in Bangladesh, $89 \%$ of women with HDPs had their BP measured on the first day after giving birth, but again by the fifth day BP was only checked in $16 \%$ of women [16]. In Nigeria, the BP of women with chronic hypertension remained high (71\%) one year after childbirth. However, 38\%, 36\%, and $16 \%$ of women with gestational hypertension, pre-eclampsia, and eclampsia, respectively, also remained hypertensive [15].
Women at risk of developing postnatal hypertension (Smith et al 2013)

- Preterm delivery triggered by maternal hypertensive disease

- Hypertension requiring antenatal treatment

- Severe antenatal hypertension (>160/110mmHg)

- Antenatal pre-eclampsia $>75 \%$

\section{WHAT IS THE POTENTIAL IMPACT OF IMPLEMENTING PROMISING BEST PRACTICES?}

- Early detection and management of hypertension in the postnatal period results in timely treatment and follow up

- Women are more aware of the long-term health implications resulting in improved health literacy

- Women with non-communicable disease may be better prepared for a future pregnancy

- Potentially expediated reduction in maternal morbidity and mortality 


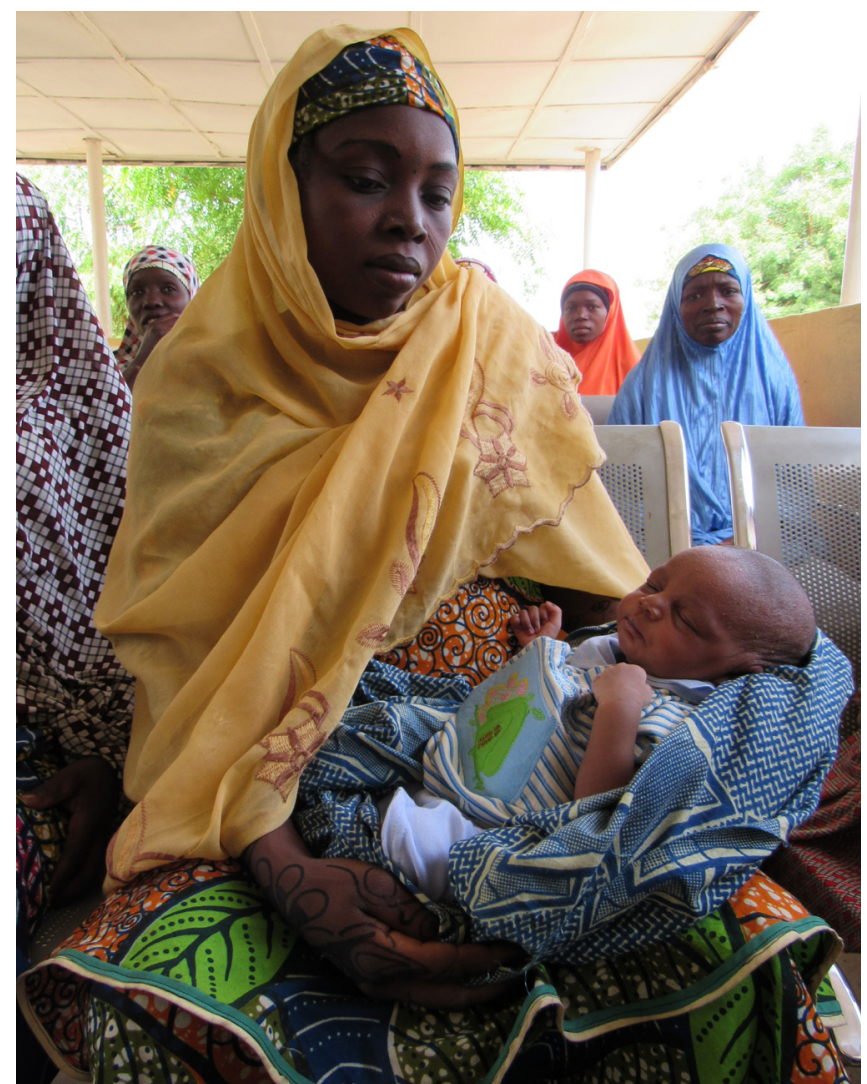

\section{HOW TO DO IT: TIPS FROM IMPLEMENTATION EXPERIENCE}

Based on programmatic experience, the following strategies can help facilitate successful monitoring of $\mathrm{BP}$ in the extended postnatal period to improve early detection and management of hypertension.

- Consider the context and level of health facility and skills of different cadres. Recognize skills mix of available health care providers. Community health workers (CHWs) can provide information to women and families on postnatal complications. Lower level providers (including $\mathrm{CHWs}$ ) are capable of measuring $\mathrm{BP}$, and primary health care $(\mathrm{PHC})$ providers can manage/refer women with hypertensive disorders in the postnatal period (including administration of $\left.\mathrm{MgSO}_{4}\right)$.

\section{POSTNATAL PERIOD (0-6 WEEKS)}

- Women with HDPs should be automatically referred to physician/specialist for follow-up

- Review international guidelines on HDPs in PNC and adapt country level guidelines and protocols for health workers. Follow WHO recommendations for PNC contacts: measure BP immediately after birth, 3-7 days, 2 weeks and 6 weeks after birth and any future contact with the health system $[1,2]$

- PNC should be an ongoing process rather than a single encounter, and all women should have contact with health system within the first three weeks postnatal and beyond $[1,14,17]$

- Develop PNC protocols for all recommended contacts and ensure protocols include monitoring of BP and management of hypertension

- If there is no Group ANC model in place, consider extending to Group PNC - this provides peer support to continue to monitor BP into the extended PNC period. If no Group ANC consider initiating a "Mothers and Baby's club" at community level.

\section{EXTENDED POSTNATAL PERIOD (6 WEEKS TO 12 MONTHS)}

- After 6 weeks, use every contact that a woman has with the health system to reduce missed opportunities to check their BP. Such instances include when bringing their baby for check-ups, growth monitoring, immunizations and for postnatal family planning.

- Integrate services with primary care to ensure seamless transition for care throughout the life course. At any facility contact beyond 6 months monitor BP if previously high and referral to physicians. It is important to consider continuous family care or life cycle approach [18]

\section{PATIENT LITERACY}

- Counsel women treated for HDPs about increased risk of future heart disease. Advise women about future pregnancy risks and health and the need for routine BP checks.

- Challenges in health-seeking behavior in the postnatal period need to be addressed. Women with pregnancy and postnatal complications and their families require more information to enable them to be aware of potential future health complications and why they should attend follow-up consultations and ask for BP checks.

- Identify opportunities to discuss with communities the risks in future pregnancies and long-term health risks for women with HDPs, and find ways to bring them for follow-up or how to increase efficiency of the service providers for effective counseling. 
Recommendations on measuring BP and managing hypertension postnatally.

Measure BP after birth, [1,2] and continued daily till discharge [1].

- Provide close postnatal follow up until BP continues to be normal/returns to normal or is managed appropriately.

- Treat women with antihypertensive drugs in the postnatal period if they: (1) have severe hypertension; or (2) were treated with antihypertensive drugs during pregnancy [2]. Keep BP at less than $160 / 110 \mathrm{mmHg}$. Further investigation is required if hypertension persists beyond three months [1]. (ISSHP 2018).

- Review women's risk factors on discharge (and at subsequent PNC contacts), and inform women with HDPs of the risk of recurrence in future pregnancies [8] and future cardiovascular risks [12].

- Review all women at 3 months postnatal by which time BP, urinalysis, and laboratory tests should have normalized [1]. If these indicators haven't normalized, refer to physicians as appropriate.

- Develop and strengthen postnatal policy/protocols to ensure women who experienced pre-eclampsia/ eclampsia are followed up. This includes providing a client held "HDP card" to record her BP and other tests during the extended postnatal period.

\section{FACTORS THAT INHIBIT SUCCESS}

- Policy factors: There are notable inconsistencies on when to measure BP and how to manage hypertension in both the immediate and extended postnatal period at global and national levels [19].

- Structural drivers: Poor procurement processes; one BP cuff shared between ANC, labor and delivery and other departments; and poor quality of BP measurement tools, including a lack of validated BP measurement machines, cuffs and stethoscope; limited awareness of all cadres of health providers on importance of routine BP measurement; limited skills updates; staff shortages; and frequent turnover.

- Poor coordination or lack of teamwork between PHC level, midwives, obstetricians, and physicians, resulting in women getting lost in the system.
- There is a lack of policy guidelines for task sharing related to measuring and managing high BP, conducting comprehensive care, and treating hypertension, including administering loading dose of $\mathrm{MgSO}_{4}$ and antihypertensives.

- Women leave health facilities soon after birth and do not complete recommended PNC contacts (including BP measurement and management) due to lack of awareness of risks.

\section{PRIORITY RESEARCH AREAS}

- What is driving the increase in postnatal pre-eclampsia/ eclampsia?

- Linking pre-eclampsia and gestational hypertension to NCDs in lower middle income countries: long term effect and survival re women getting the right care; maternal mental health (postpartum depression, anxiety, and PTSD) linked to HDPs.

- How to best deliver postpartum cardiovascular risk reduction programs in lower middle income countries

- What is the ideal duration of $\mathrm{MgSO}_{4}$ as an anticonvulsant postnatally?

- Large longitudinal cohorts to embrace "Fourth Trimester" using a life course perspective

- Evaluate intervention and review plans for scale up and scalability

\section{INDICATORS FOCUSING ON PRE- ECLAMPSIA AND BP: FROM WHO STANDARDS OF MNH QOC}

- The proportion of all women in PNC wards or areas in the health facility who have documented problems of BP, pulse rate, vaginal bleeding, lochia or breastfeeding. (84.02)

- The health facility has functioning essential equipment and supplies for the detection of complications (e.g. thermometers, sphygmomanometers, fetal stethoscopes, urine dipsticks, pulse oximeter) in sufficient quantities at all times in the labour and childbirth areas of the maternity unit. (91.63)

- The health facility has written, up-to-date clinical protocols on the management of pre-eclampsia that are available in the labour, childbirth and postnatal areas and are consistent with WHO guidelines. (90.59) 
- Health-care staff in the maternity unit receive inservice training and regular refresher sessions in the use of antihypertensive agents, intravenous infusion and $\mathrm{MgSO} 4$ for treating pre-eclampsia and eclampsia at least once every 12 months. (84.54)

- The proportion of all women discharged postpartum within the past 24 hours had an accurately completed record of processes of care, treatments, outcomes and diagnoses (with ICD code). (82.88)

- The health facility has supplies of antihypertensive agents and $\mathrm{MgSO}_{4}$ (and the antidote calcium gluconate) in sufficient quantities, available at all times, in antenatal, labour, childbirth and postnatal areas for the management of women with preeclampsia. (92.81)

\section{TOOLS AND RESOURCES}

- FIGO https://www.figo.org/preeclampsia-guidelines

- ISSHP Classification, Diagnosis, and Management Recommendations for International Practice

- Nathan HL, Duhig K, Hezelgrave NL, Chappell LC, Shennan AH. Blood pressure measurement in pregnancy. The Obstetrician \& Gynaecologist 2015; 17:91-8.

- NICE Hypertension in pregnancy: diagnosis and management guideline [NG133] NHS UK 2019

- World Health Organization. WHO recommendations for the prevention and treatment of pre-eclampsia/ eclampsia. Geneva; 2011 http://apps.who.int/iris/ bitstream/10665/44703/1/9789241548335_eng. pdf

- World Health Organization. WHO recommendations on postnatal care of the mother and newborn. Geneva, 2013 http://www.who.int/maternal_ child_adolescent/documents/postnatal-carerecommendations/en/.

- WHO 2016 Standards for improving quality of maternal and newborn care in health facilities WHO 2017 Managing Complications in Pregnancy and Childbirth (MCPC): A Guide for Midwives and Doctors second edition.

- WHO Integrating Management of Pregnancy and Childbirth (IMPAC): 2017 Consider highlighting aspects of IMPAC related to integration with immunization/child health services related to reducing missed opportunities for women's checkups in the postnatal period.

\section{REFERENCES}

1. Brown M, Laura A. Magee, Louise C. Kenny, et al, on behalf of the International Society for the Study of Hypertension in Pregnancy (ISSHP) Hypertensive Disorders of Pregnancy: ISSHP Classification, Diagnosis, and Management Recommendations for International Practice Hypertension. 2018; 72:24-43

2. World Health Organization (WHO) Recommendations for prevention and treatment of pre-eclampsia and eclampsia, World Health Organization 2011. Available at Website: https://apps.who.int/iris/bitstream/ handle/10665/44703/9789241548335_eng.pdf Accessed 29 Dec. 2019

3. Bramham Kate, Nelson- Piercy Catherine, Brown Morris J, Chappell Lucy C. Postpartum management of hypertension BMJ 2013

4. Deruelle P, Coudoux E, Ego A, Houfflin-Debarge V, Codaccioni X, Subtil D. Risk factors for post-partum complications occurring after preeclampsia and HELLP syndrome. A study in 453 consecutive pregnancies. Eur J Obstet Gynecol Reprod Biol 2006; 125(1):59-65

5. Royal College of Obstetricians \& Gynaecologists. Greentop Guideline 37a: Reducing the risk of thrombosis and embolism during pregnancy and the puerperium. 2015; Available at: https://www.rcog.org.uk/en/guidelinesresearch-services/guidelines. Accessed Jul/19, 2015.

6. Firoz T, Melnik T. Postpartum evaluation and longterm implications. Best Pract Res Clin Obstet Gynaecol. 2011;25(4):549-561. doi: 10.1016/j. bpobgyn.2011.03.003

7. Firoz T, Sotunsa J. Adetoro 00. Treatment postpartum - immediate and long term. In: The Federation of Gynecology and Obstetrics (FIGO) Textbook of Pregnancy Hypertension. Eds. Magee LA \& von Dadelszen P. The Global Library of Women's Medicine, London, 2016, pp 215-232.https://www.glowm.com/resource_type/ resource/textbook/title/the-figo-textbook-of-pregnancyhypertension/resource_doc/2768

8. NICE Hypertension in pregnancy: diagnosis and management guideline [NG133] NHS UK 2019

9. Benova L, Owolabi O, Radovich E, Wong KLM, Macleod D, Langlois EV, et al. (2019) Provision of postpartum care to women giving birth in health facilities in sub-Saharan Africa: A cross-sectional study using Demographic and Health Survey data from 33 countries. PLoS Med 16(10): e1002943. https://doi.org/10.1371/journal. pmed.1002943 
10. Goel A, Rana S. Angiogenic factors in preeclampsia: potential for diagnosis and treatment. Curr Opin Nephrol Hypertens. 2013;22(6):643-650 doi:10.1097/ MNH.Ob013e328365ad98

11. Magee LA, Sharma S, Nathan HL, Adetoro O0, Bellad MB, Goudar S, Macuacua SE, Mallapur A, Qureshi $\mathrm{R}$, Sevene E, Sotunsa J. The incidence of pregnancy hypertension in India, Pakistan, Mozambique, and Nigeria: A prospective population-level analysis. PLoS medicine. 2019 Apr 12;16(4): e1002783.

12. Smith, Marie., Jason Waugh, Catherine Nelson Piercy Management of postpartum hypertension 2013. The Obstetrician \& Gynaecologist. http://onlinetog.org https://doi.org/10.1111/j.1744-4667.2012.00144

13. Melchiorre K, Sutherland GR, Liberati M, Thilaganathan B. Preeclampsia is associated with persistent postpartum cardiovascular impairment. Hypertension 2011 Oct;58(4):709-715.

14. World Health Organization. WHO recommendations on postnatal care of the mother and newborn. Geneva, 2013 http://www.who.int/maternal_child_adolescent/ documents/postnatal-care-recommendations/en/.

15. Warren CE, Ishaku SM et al, Landscape Analysis of Preeclampsia/Eclampsia in Nigeria Report. New York, USA: Population Council; 2016
16. Hossain S, Roy S, Sultana K, Warren CE. Assessing the effect of a primary health care intervention for improving pre-eclampsia and eclampsia knowledge and practice in Bangladesh. New York USA: Population Council; 2019.

17. ACOG Practice Bulletin No. 202/203. Gestational hypertension and preeclampsia. American College of Obstetricians and Gynecologists. Obstet Gynecol 2019;133: e1-25.

18. Arabin, Birgit and Ahmet A. Baschat. Pregnancy: An Underutilized Window of Opportunity to Improve Long-term Maternal and Infant Health-An Appeal for Continuous Family Care and Interdisciplinary Communication Front. Pediatr. 5:69. doi: 10.3389/ fped.2017.00069

19. Gillon TE, Pels A, von Dadelszen P, MacDonell K, Magee LA. Hypertensive disorders of pregnancy: a systematic review of international clinical practice guidelines. PLoS One. 2014;9(12):e113715. Published 2014 Dec 1. doi:10.1371/journal.pone.0113715

Acknowledgments: This document was originally drafted by Charlotte Warren. Critical review and helpful comments were provided by Deborah Armbruster, Tabassum Firoz, Emily Hillman, Sharif Hossain, Salisu Ishaku, Karen Kirk, Tracy McClair, Pooja Sripad, and Catherine Taylor.

For further information please contact us at MNH-PCDC@popcouncil.org

Suggested citation: High-Impact Practices (HIPs) in Maternal and Newborn Health. Detecting and managing hypertension in the extended postnatal period: Supporting women to be at the center of their own care as active participants. Washington (DC): Population Council; 2020. Available from: https://www.popcouncil.org/research/ending-eclampsia1

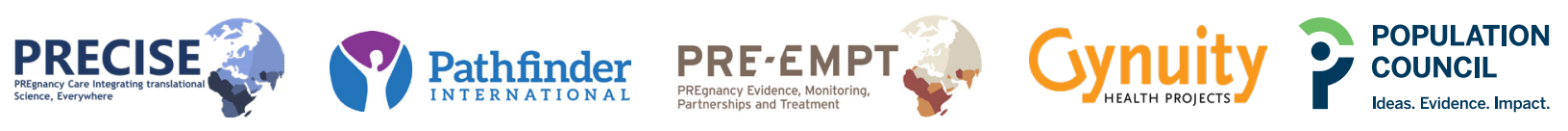

